\title{
Beam Beam Tune Shifts for 36 Bunch Operation in the Tevatron
}

\author{
Peter Bagley \\ Fermi National Accelerator Laboratory \\ P.O. Box 500, Batavia, Illinois 60510
}

October 1996

Presented at EPAC '96, 5th European Particle Accelerator Conference, Sitges (Barcelona), Spain, June 10-14, 1996. 


\section{Disclaimer}

This report was prepared as an account of work sponsored by an agency of the United States Government. Neither the United States Government nor any agency thereof, nor any of their employees. makes any warranty, express or implied. or assumes any legal liability or responsibility for the accuracy. completeness or usefulness of any information, apparatus, product or process disclosed, or represents that its use would not infringe privately owned rights. Reference herein to any specific commercial product, process or service by trade name, trademark, manufacturer or otherwise, does not necessarily constitute or imply its endorsement, recommendation or favoring by the United States Government or any agency thereof. The views and opinions of authors expressed herein do not necessarily state or reflect those of the United States Government or any agency thereof.

\section{Distribution}

Approved for public release: further dissemination unlimited. 


\title{
CONF-96/340 \\ BEAM BEAM TUNE SHIFTS FOR 36 BUNCH OPERATION IN THE TEVATRON
}

\author{
Peter Bagley, Fermilab*, P.O. Box 500, Batavia, Ill., USA, 60510
}

\section{Abstract}

We are preparing to upgrade the Tevatron Collider from 6 to 36 bunch operation. The 36 bunches are in 3 "trains" of 12 bunches. The spacing between bunches within a train is $21 \mathrm{RF}$ buckets (53.106 MHz) and 139 empty buckets separate the trains. Because the 36 bunches are not evenly spaced around the machine, the different bunches within a train pass the opposing bunches at different points in the ring and so feel different beam beam effects. Through most of the machine the beams have helical separation, so these are mainly long range beam beam effects. As a first, very simple step, we've looked at the differences in the tunes of the different anti-proton (pbar) bunches. During the 36 bunch studies in Fall 1995, we used a new tune measurement system to measure these in several different machine conditions. We compare these measurements to calculations of the tunes for a pbar with zero transverse and longitudinal oscillation amplitudes. We discuss experimental problems, and the assumptions, approximations, and effects included in the calculations. Our main intent is to gain confidence that we can accuratcly model beam beam effects in the Tevatron.

\section{INTRODUCTION}

In Run Ia and Ib, we have used 6 bunches per beam in the Tevatron. In collision conditions, with the separated orbits, each bunch sees 2 head on collisions, one at B0 and one at D0, and 10 "near misses". At $900 \mathrm{GeV}$, the diagonal separation in the arcs is greater than about 2.5 $\mathrm{mm}$, corresponding to roughly 7 sigma center to center separations.

For Run II we plan to go to 36 bunches per beam. We will still have two interaction regions, but we will have 7 times more "near misses", 70 instead of 10 .We are concerned about the increased importance of the many "near miss" beam beam effects. There are many aspects to this problem. The near miss beam beam interactions will change the orbits, tunes, coupling, dispersion, and $\beta^{*_{1}}$ s of the bunch as a whole and as a function of the individual particle's amplitudes. Also the non-linearity of the separated beam beam interaction will drive resonances, including many resonances not driven (or only driven at higher order) by the head on beam beam interaction.

In 36 bunch mode, the bunches are not evenly distributed around the ring. Because of this, all the bunches do not see the near misses at the same locations in the ring and consequently these beam beam effects can

*Operated by the Universities Research Association, Inc. under contract with the U.S. Department of Energy. be different for the different bunches.

In this paper we will only look at one aspect of the problem, the tune shifts for the individual bunches and consequently the spread in the tunes of the different pbar bunches. This is only one small simple piece of the problem, but it is a start at looking at the beam beam effects and it is something that we can directly measure in the machine and compare to our calculations.

\section{TUNE SHIFT CALCULATION}

For the tune shift of a bunch, we will calculate the tune shift of a particle with zero horizontal and vertical betatron amplitudes and with zero energy offset. We start from the beam beam potential [1]

$U(x, y)=\frac{N r}{\gamma} \int_{0}^{\infty} d q \frac{1-\exp \left[\frac{-x^{2} / \sigma_{x}^{2}}{2(1+q R)}+\frac{-y^{2} / \sigma_{y}^{2}}{2(1+q / R)}\right]}{\sqrt{(1+q R)(1+q / R)}}$

where $N$ is the number of particles in the opposing bunch, $\gamma$ is the relativistic factor, $r$ is the classical radius of the proton, $1.535 \times 10^{-18} \mathrm{~m}$, and $R$ is the aspect ratio of the opposing beam, $\left(\sigma_{y} / \sigma_{x}\right)$. The $x$ and $y$ are the displacements of the test particle from the center of the opposing beam on each turn. The beam beam kicks given to a particle on each turn are then $\Delta z^{\prime}=-\partial U / \partial z$. where $z$ may stand for either $x$ or $y$.

Using the forms $\left(x=x_{O}+\delta x\right)$ and $\left(y=y_{O}+\delta y\right)$, where $x_{O}$ and $y_{o}$ are constants, the tune shifts for a zero amplitude particle are

$$
\xi_{z}=\frac{-\beta_{z}}{4 \pi}(k l)_{z}=\frac{-\beta_{z}}{4 \pi}\left(\frac{\partial\left(\Delta z^{\prime}\right)}{\partial(\delta z)}\right)
$$

For a given Tevatron condition (energy, lattice, and collision or injection helix), we run the program MAD [2] for each beam to calculate the twiss parameters for each beam and the separations between the beams at the 72 locations where that pbar bunch will encounter the opposing proton bunches. (These separations are modified slightly by the average beam beam kicks. We include this small effect in our calculations.)

We combine these with measured or assumed intensitics, emittances and energy spreads and do a numerical integration of the above equations for $\xi_{x}$ and $\xi_{y}$. We simply add the $\xi_{x}$ and $\xi_{y}$ from each crossing point to get the total horizontal and vertical tune shifts for the pbar bunch. (This is an approximation to first order in the $\xi_{x}$ and the $\xi_{y}$ from each crossing point.) There is also a skew quad component to the beam beam kick. For our conditions, this has only a small effect on the global 
coupling, the minimum tune split. Although we compute this, we will not discuss it in this paper.

\section{BUNCH MODE IN THE TEVATRON}

For 36 bunch running in the Tevatron, we plan to run with 3 bunch "trains" with 12 bunches in each train. The harmonic number of the Tevatron is 1113 . Within a "train", the bunches will have 21 buckel (395. nsecs) spacing and separating the trains will be an abort gap of 139 emply buckets $(2.617 \mu \mathrm{secs})$.

Many of the near misses seen by a bunch will be $21 / 2$ buckets $(59.3 \mathrm{~m})$ apart. By a curious coincidence, the standard cell length in the Tevatron is $59.5 \mathrm{~m}$, very nearly the same as the spacing hetween near misses. Most of the near misses seen by a bunch will be at the same location within the standard cell

We spend the most time in the injection and the collision conditions and so these two conditions most concern us. Both the transverse separation between the beams, that is the helix, and the longitudinal timing of the beams, that is the cogging, are different in the injection and collision conditions.

Our standard parameters for the upcoming Run II are proton intensities of $300 \mathrm{e} 9 /$ bunch, pbar intensities of $60 \mathrm{e} 9 / \mathrm{bunch}$, emittances of $20 \pi \mathrm{mm} \mathrm{mrad}$, and fractional momentum spreads of $0.15 \mathrm{e}-3$ at $900 \mathrm{GeV}$.

With these parameters, in our collision conditions, the $\xi$ 's from each near miss in the arcs are small, typically less than 0.001, and they largely cancel. However, the first near misses on either side of the interaction points are an exception. At these points the separation between the beams is only about half what it is in the arcs and one of the $\beta$ 's is large. These points contribute a total of 0.010 to $\xi_{x}$ and $\xi_{y}$. Further, the first pbar bunch in each train does not meet with a proton bunches at the first near miss before the interaction point. As a result, its total $\xi y$ is about 0.01 lower than that of the other bunches. Similarly, the total $\xi_{x}$ of the last pbar bunch in each train is about 0.01 lower than that of the other bunches. We are concerned about these differences.

Excluding the contributions from the head on collisions at the interaction points and these first near misses, the magnitudes of the total $\xi_{x}$ and $\xi_{y}$ are smaller than 0.003 .

\section{EXPERIMENTAL METHOD}

During the 36 bunch machine studies periods in Nov. 1995 and in Feb. 1996, we made a series of measurements of the pbar beam beam tune shifts. We took data in several machine configurations, but for brevity, we will only show 2 conditions in this paper.

We used $36 \times 1$ stores, that is 36 proton bunches and one pbar bunch. Twelve proton bunches are injected, accelerated, and coalesced during a single Main Ring cycle. There were problems coalescing 12 bunches at a time in the Main Ring. These resulted in large satellites (large numbers of protons in the RF buckets just before and just after the "main" or intended bucket) and low proton intensities of about a third of what we achieve when coalescing only a single proton bunch at a time. Typical bunch by bunch proton intensities and $95 \%$ emittances can be seen in Fig. 1. The large emittances for proton bunches 1,13 , and 25 are due to ringing in the pbar injection kicker.

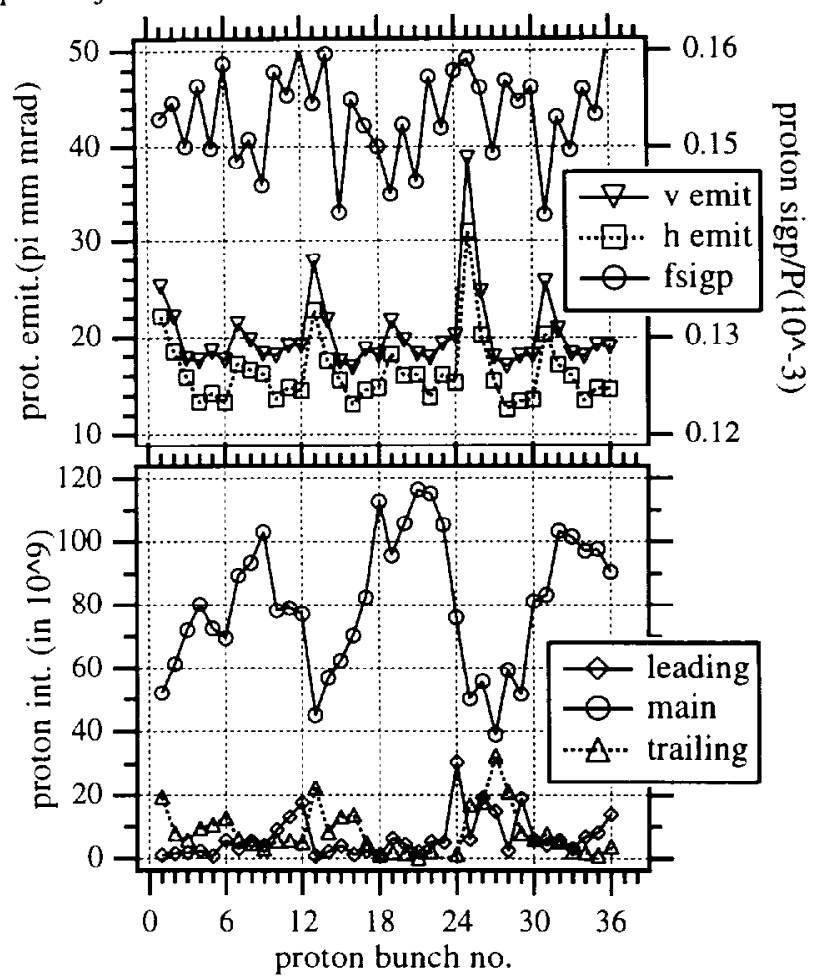

Figure 1: Bunch by bunch proton intensities and emittances at $900 \mathrm{GeV}$ in colliding beam conditions.

Once our single pbar bunch was injected and we were in the machine configuration for our measurements, we would identify the proton and pbar tunes, globally decouple the pbar tunes and leave the pbar tunes separated by several times the minimum tune split. (The point of these measurements is to look at the beam beam tune shifts. We set up our conditions to make us insensitive to the changes in the beam beam coupling.) Also we made sure that the proton and the pbar tunes were separated and distinct from each other.

We then cogged the single pbar bunch into the timing positions occupied by the 36 different pbar bunches. Changing the pbar cogging, that is the pbar timing relative to the protons, determines where it will encounter the proton bunches.

We used two different systems to measure the tunes of the pbar bunch. Dave McGinnis and John Marriner have set up a system at the F0 location using the Tevatron "superdampers" and new front end electronics to process the signals. This system includes fast timing gates for both the noise source applied to the beam and to the 
signal from the motion of the beam. This system worked extraordinarily well. With 36 proton bunches of about $100 \mathrm{e} 9$ protons per bunch, and only one pbar bunch of about 5.e9 pbars, we could still distinguish the pbar tunes. We will refer to this as the Fo system

We also measured the pbar tunes on the standard Tevatron tune measurement system, which uses pairs of strip line pickups located at A17. Although this system did not discrimate between the protons and pbars nearly as well as the F0 system, when we used the F0 system to excite only the pbars, the pbar tunes could be seen on the A17 system.

\section{DATA AND ANALYSIS}

\subsection{Colliding Beam Conditions}

Figure 2 shows measured and calculated pbar tunes in colliding beam conditions.

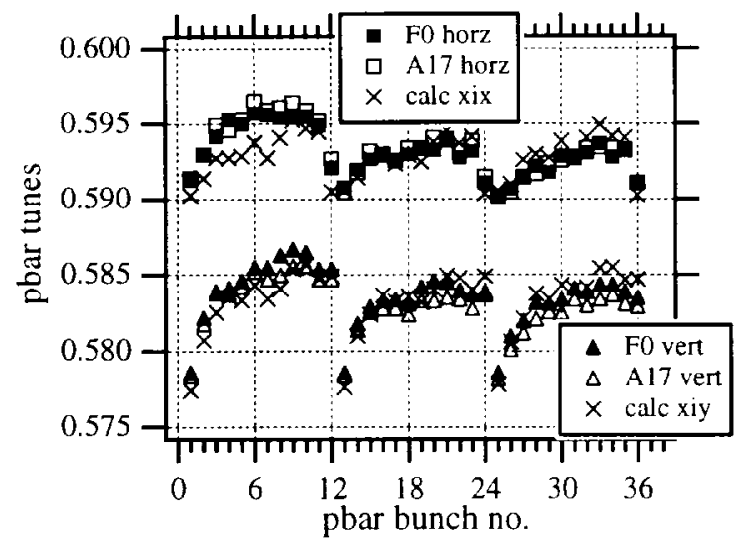

Figure 2 : Measured and Calculated Pbar Tunes for Colliding Beam Conditions. We assume base pbar horizontal and vertical tunes of 0.5855 and 0.5755 , horizontal proton emittances equal to the vertical proton emittances, and we use a scale factor of 0.648 for the tune shifts from the head on beam beam interaction.

During the several hour measurement, the proton intensitities decreased by about $3 \%$ or less, the proton emittances increased by about $2 \pi$, and the $\sigma_{p} / p$ increased by about 1.e-5. These only change our calculated $\xi$ by about \pm 0.001 .

The proton bunches often had large satellites. However, since an RF bucket is short compared to the $\beta$ 's at most locations in the ring, it makes very little difference whether all the protons are in the main bucket or if they are distributed among 3 adjacent buckets. For the proton intensities shown in Fig. 1, this changes the tune shifts by less than 0.0003 .

The measured and calculated separations due to the helix differed by about \pm 5 to $10 \%$. Modeling this as an overall scale factor on the size of the helix, the tune shifts change by less than about \pm 0.002 .

For locations where the beams are separated, the emittance has only a weak effect on the tune shift parameters. But the emittance is important at the two locations where the beams are in collision. The emittances shown in Fig. 1 come from measurements of the beam sizes from the flying wire system. This is a complicated system and we would not be surprised if there were 10 to $20 \%$ systematic errors in the emittance measurements. Because the vertical beam size is only weakly affected by the momentum spread of the beam, in our calculations we have used the measured vertical emittance for both the horizontal and vertical emittance.

When we read the tunes off the spectrum analyzers, we read a value corresponding to the center of the tune line. But we have calculated the tune shifts for zero amplitude particles. For a gaussian distribution, most particles have oscillation amplitudes of about 1 sigma and the average oscillation amplitude is 1.25 sigma. For crossing points where the beams are separated by several beam sizes, these oscillation amplitudes have little effect on the beam beam tune shifts. But if the beams are colliding head on, these oscillation amplitudes will decrease the beam beam tune shifts by a factor of 0.648 . This is an important effect for the data in Fig. 2. Although this is only a hand waving estimate of the effect of the distribution of the oscillation amplitudes of the particles, we have used this factor of 0.648 to scale the head on tune shifts in our calculations for Fig. 2.

We actually calculate only the beam beam tune shifts for the pbars. We must add to this the pbar "base tune", the pbar tune if there were no protons present. We did not measure this directly, but have chosen it "by eye".

The agreement between the measurements and the calculations in Fig. 2 is quite good. In contrast to the "ideal" parameters, most of the variation in tunes between the pbar bunches is due to the variations in the proton intensities, although we can see some evidence of the strong effects from the first near misses on either side of the head on interaction points.

\subsection{Pbar Injection Conditions}

Fig. 3 shows the measured and calculated pbar tunes for our pbar injection conditions. In this condition the beams are separated everywhere. Without the head on collisions, the pbar tune shifts are much less sensitive to the proton emittances and the correction for the peak of the tune distribution vs. the tune of a zero amplitude particle is much smaller.

Proton intensities, emittances, energy spreads are not shown but are qualitatively similar to those shown in fig. 1 for the colliding beam condition measurements.

For visual clarity, fig. 3 uses a small offset between the $\mathrm{A} 17$ and $\mathrm{F} 0$ vertical tune.

The agreement is very good, except for the first few bunches. These are the first few bunches where we took data and so we may not have been reading the tunes correctly yet. 


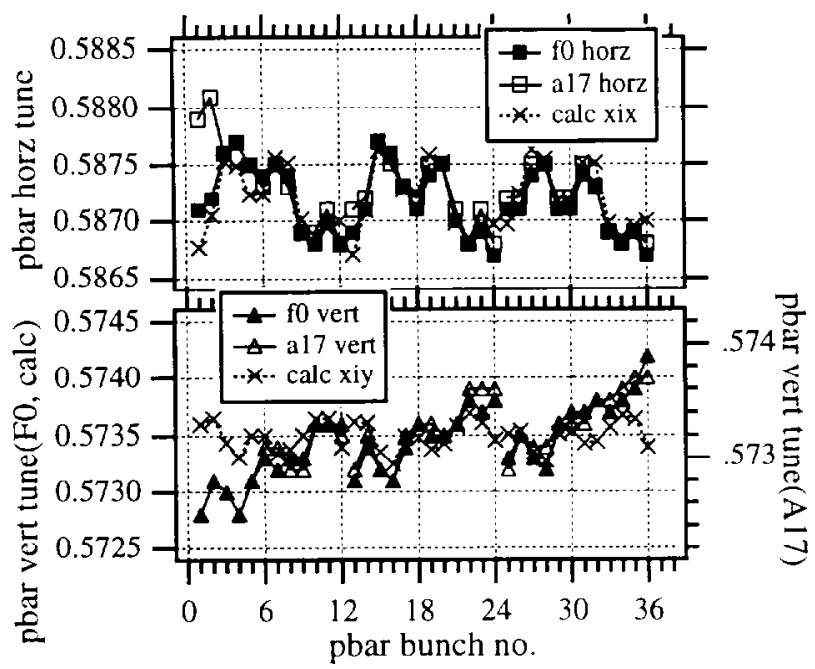

Figure 3 : Measured and Calculated Pbar Tunes for Pbar Injection Conditions. The calculations use base pbar horizontal and vertical tunes of 0.5869 and 0.5736 and , assume horizontal proton emittances equal to the vertical proton emittances. In this condition, the beams do not collide.

\section{CONCLUSIONS}

The good agreement between our calculations and the measurements has increased our confidence in our method and our models of the Tevatron.

Proton intensities for this experiment were much lower and more uneven than what we hope to have for Run II. Much of the variation in the tunes of the different pbar bunches is from this uneven proton intensity. This obscures the "outlier problem" in this data.

We still want to work on reducing the tune shifts from the first near misses. Presently we feel the most promising approach is to look at modifying the lattice to reduce the beta at that point. When we have improved this and we have a lattice and helix design that produces reasonable beam beam orbit distortions, tune shifts, and couplings, we can continue on to more involved calculations, for example more calculations of the tune shifts with particle amplitudes and of the resonance strengths and widths.

\section{REFERENCES}

[1] "Calculation of Luminosity and Bcam-Bcam Detuning in Coasting Beam Interaction Regions", B. W. Montague, CERN/ISR-GS/75-36, (1975).

[2] "The MAD Program - User's Reference Manual", Hans Grote and F. Christoph Iselin, CERN/SL/90-13 (Rev.4).

\section{ACKNOWLEDGEMENTS}

Particular thanks to Glen Goderre, Leo Michelotti, Pat Colestock, John Johnstone, and John Marriner. Most of the members of the MAD group participated in this series of measurements. 